Poster presentation

\title{
Free breathing myocardial perfusion MRI with SW-CG- HYPR using motion correction
}

\author{
Lan Ge*1, Aya Kino ${ }^{1}$, Mark Griswold ${ }^{2}$, James Carr ${ }^{1}$ and Debiao Li ${ }^{1}$
}

Address: ${ }^{1}$ Northwestern University, Chicago, IL, USA and ${ }^{2}$ Case Western Reserve University, Cleveland, OH, USA

* Corresponding author

from 13th Annual SCMR Scientific Sessions

Phoenix, AZ, USA. 21 -24 January 2010

Published: 21 January 2010

Journal of Cardiovascular Magnetic Resonance 20 I0, I 2(Suppl I):P205 doi:10.1 I86/1532-429X-I2-SI-P205

This abstract is available from: http://jcmr-online.com/content/I2/SI/P205

(C) $2010 \mathrm{Ge}$ et al; licensee BioMed Central Ltd.

\section{Introduction}

The diagnostic value of first-pass perfusion MRI is limited by the low spatial coverage, resolution, SNR, and motion artifacts. Sliding-Window Conjugate-Gradient HYPR [1] (SW-CG-HYPR) has been proposed to acquire perfusion images with increased spatial coverage, better spatial resolution, and improved SNR [2]. However, this method is sensitive to the respiratory motion and thus limited to the breath hold. Motion correction may be useful to reduce motion artifacts and allow for free-breathing first-pass perfusion.

\section{Purpose}

To develop and test a non-model-based motion correction method combined with SW-CG-HYPR to perform free-breathing myocardial MR imaging.

\section{Methods}

An ECG-triggered, multi-slice FLASH sequence with radial sampling was used. As shown in Figure 1, radial sampling was applied in a segmented interleaved fashion. Multiple slices were acquired after each saturation recovery prepulse. The motion correction method is illustrated in Figure 2. Both translation and rotation of the heart were detected in image domain by calculating the normalized cross-correlation coefficients. Motion correction was performed in $k$-space by rotating the undersampled $k$-space and shifting the phase by a factor of $\exp \left(-2 \pi \mathrm{i}\left(\delta_{\mathrm{x}} / \mathrm{N}_{\text {read }}+\delta_{\mathrm{y}} /\right.\right.$ $\left.\mathrm{N}_{\mathrm{pe}}\right)$ ), where $\delta_{\mathrm{x}}$ and $\delta_{\mathrm{y}}$ are the number of pixels to shift in $x$ and $y$ direction, and $\mathrm{N}_{\text {read }}$ and $\mathrm{N}_{\mathrm{pe}}$ are the total number of pixels along readout and phase encoding direction. Sliding window was used to reconstruct the composite images, and the time-resolved images were reconstructed after CG-HYPR processing. Six healthy volunteers were scanned using a $1.5 \mathrm{~T}$ system, with and without breath hold, during first-pass of the contrast agent. Imaging parameters included: TR/TE/flip-angle $=3.2 / 1.6 \mathrm{~ms} / 10^{\circ}$, spatial resolution $=1.3 \times 1.3 \times 10 \mathrm{~mm}^{3}$, and number of slices $=6$. The images were qualitatively graded by $\mathrm{a}$ reviewer using a score of 1-4 (1: worse; 4 : best), and the signal changes vs. time curves were compared.

\section{Results}

The average image quality score of the free-breathing images with motion correction $(3.09 \pm 0.37)$ is significantly higher than those without motion correction (2.26 $\pm 0.40)$, and is comparable to the successful breath-holding images $(3.10 \pm 0.41)$ (Figure 3$)$. The signal changes in motion corrected free-breathing images were closely related to those in the breath-holding images, with a correlation coefficient of 0.9764 for myocardial signals (Figure 4 and 5).

\section{Conclusion}

The image quality of myocardial perfusion MRI using SWCG-HYPR was substantially improved after motion correction. This technique may allow myocardial perfusion MRI during free breathing. 


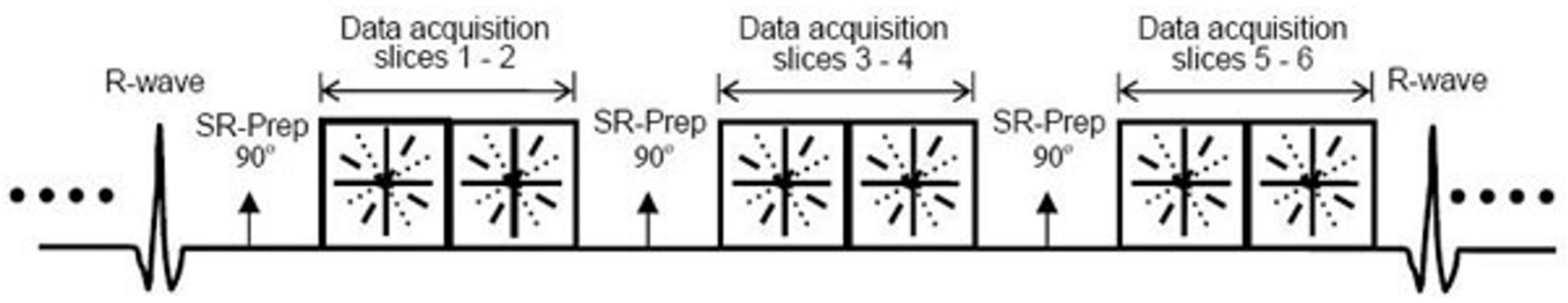

Figure I

Schematic of the myocardial perfusion acquisitions sequence. Radial $k$-space is highly undersampled, interleaved and equally space. Mulitple slices are acquired after each SR preparation pulse. In this work, two slices were acquired after each SR pre-pulse, and totally six slices were acquired for each cardiac cycle.

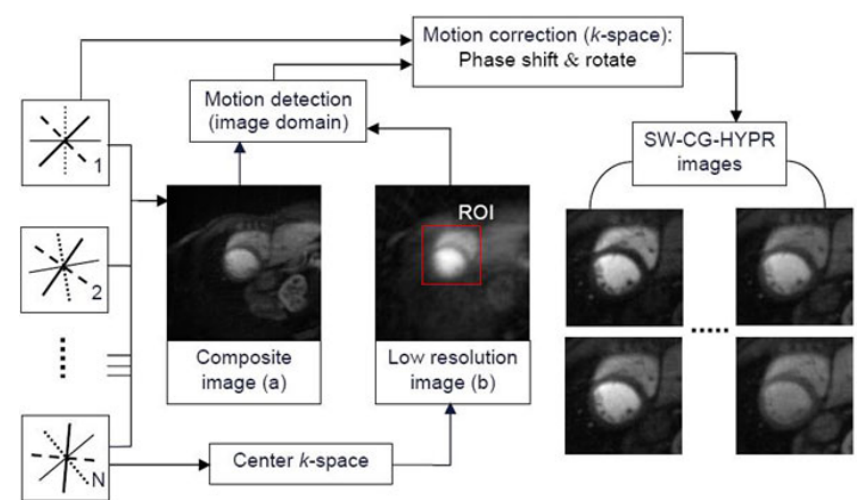

\section{Figure 2}

Schematic diagram illustration the motion correction scheme. The ROI (illustrated by the red rectangular) of the low resolution imates (b), reconstructed from the center of the undersampled $k$-space, is compared with the composite image (without motion correction) (a) for the motion detection. After the motion correction the k-space is the precessed by SW-CG-HYPR method for the timeresolved myocardial perfusion images.

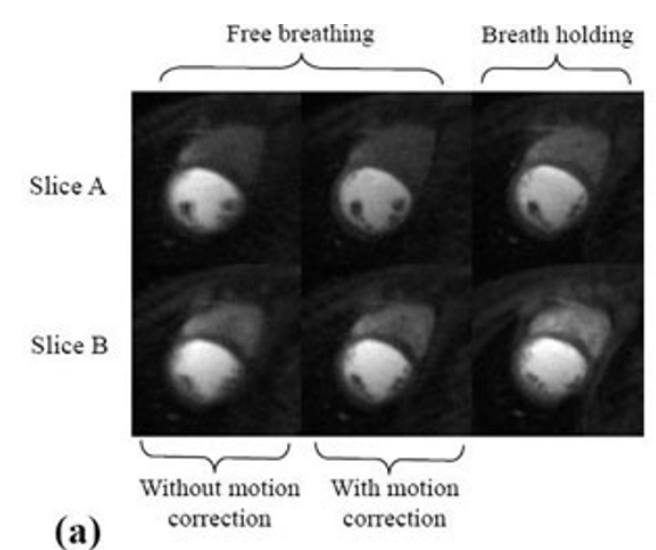

(a)

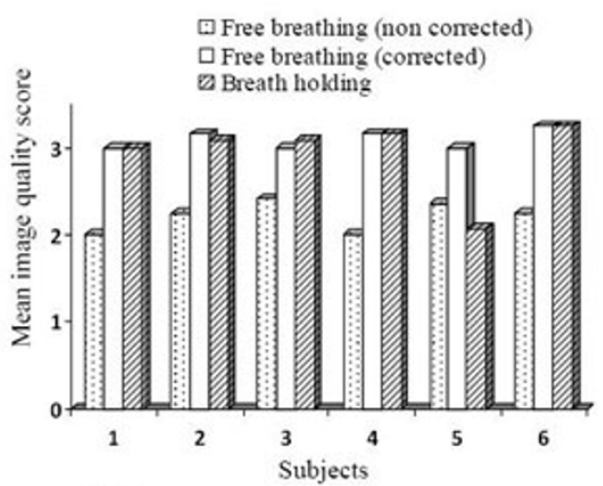

(b)

Figure 3

Comparision example (a) and mean image quality scores (b) of free-breathing images without motion correction, free-breathing images with motion correction and breath-holding images. 


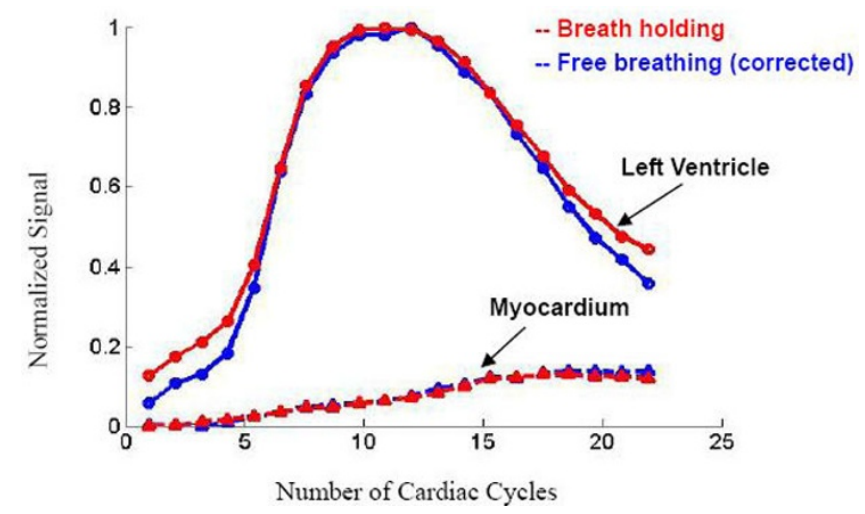

Figure 4

One example comparion of left ventericular and myocardial signal intensity changes between freebreathing images with motion correction and breath-holding images during first-pass perfusion. A close correlation between the two datasets is observed.

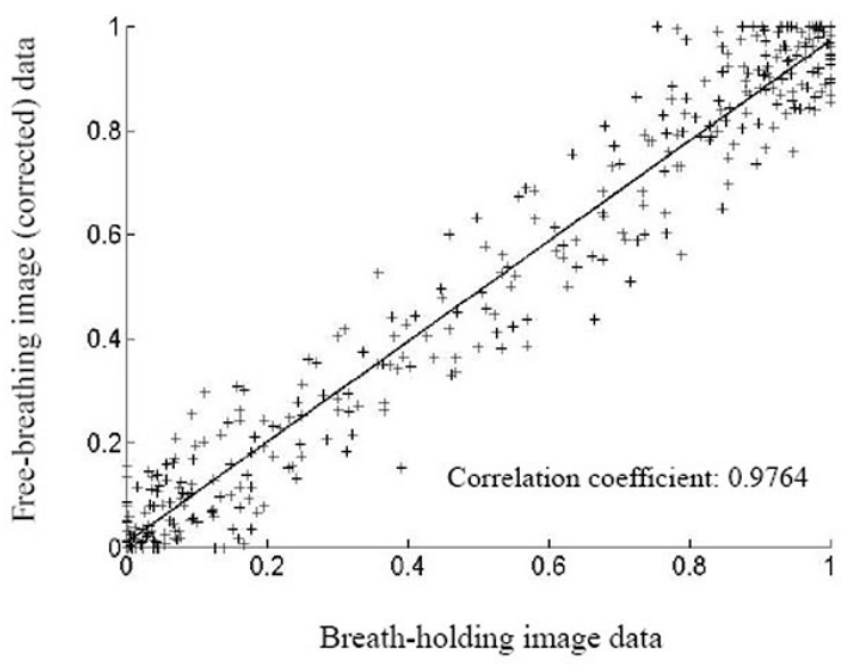

Figure 5

Correlation between signal intensities of free-breathing images with motion correction and those breathholding images for all of the volunteers. The two datasets are highly correlated, with a correlation coefficient of 0.9764

\section{References}

I. Griswold MA, et al:: \#834. Proceedings of ISMRM 2007.

2. Ge L, Li D, et al.: Magn Reson Med 2009. DOI: 10.1002/mrm.22059 